\title{
Preface to Guidelines: Laparoscopic treatment of ventral and incisional hernias by the International Endohernia Society (IEHS)
}

\author{
Alfred Cuschieri
}

Received: 27 June 2013/ Accepted: 24 July 2013/Published online: 6 September 2013

(C) Springer Science+Business Media New York 2013

Several months ago Dr. R. Bittner, as the European Editor, approached me with the request that Surgical Endoscopy publish the "Guidelines on the Laparoscopic Treatment of Ventral and Incisional Hernia" that was prepared by the International Endohernia Society (IEHS). I was quite receptive to this request for several cogent reasons: (1) the authorship represents the best known and respected international experts on hernia surgery, (2) the methodology used was solid, soliciting two experts who were assigned a specific topic with related search questions for each of the systematic reviews, and (3) the process had been transparent, culminating in confirmation by a consensus conference held at an international meeting of the IEHS involving all participants together with the international audience. There was a problem, however. The document was far too detailed and lengthy for journal publication. Therefore, after consultation with his coauthors, Dr. Bittner agreed to reduce the document by half, agreeing to publication of some of the material only in the online version of
Surgical Endoscopy. The abridged version was completed in a few weeks. As editor and objective reviewer, I consider the result to be excellent as now the guidelines document is more focused without loss of substance and with minimal repetition, which is inevitable in a multi-author document containing several systematic review chapters.

Part I of the guidelines is published in the current issue of Surgical Endoscopy and parts II and III will follow in the next issue. All three parts constitute an outstanding seminal account of evidence-based laparoscopic treatment of ventral and incisional hernia. The guidelines will, I am sure, serve as a ground-breaking document, not just for practicing surgeons, but also for hospital administrators and health technology experts. I congratulate the IEHS, and especially Dr. Reinhard Bittner, for producing this seminal work. I can testify to the many complimentary e-mails of thanks sent to him (and copied to me) from his fellow authors for achieving what they considered an almost impossible goal.

\footnotetext{
A. Cuschieri $(\bowtie)$

Institute for Medical Science and Technology, University of Dundee, 1 Wurzburg Loan, Dundee DD2 1FD, UK

e-mail: a.cuschieri@dundee.ac.uk
}

\section{A. Cuschieri}

Scuola Superiore Sant'Anna, Pisa, Italy 